\title{
Cardiovascular and cerebrovascular responses to lower body negative pressure in type 2 diabetic patients
}

\author{
Harald Marthol $^{\text {a, },}$, Udo Zikeli ${ }^{\text {a, }}$, Clive Martin Brown ${ }^{\text {b }}$, Marcin Tutaj ${ }^{\text {c }}$, Max Josef Hilz ${ }^{\text {a,d,* }}$ \\ a Department of Neurology, University of Erlangen-Nuremberg, Schwabachanlage 6, D-91054 Erlangen, Germany \\ ${ }^{\mathrm{b}}$ Division of Physiology, University of Fribourg, Rue du Musee 5, 1700 Fribourg, Switzerland \\ ${ }^{\mathrm{c}}$ Department of Neurology, Jagiellonian University, Botaniczna 3, 31503 Krakow, Poland \\ ${ }^{\mathrm{d}}$ Departments of Neurology and Medicine, New York University, 550 First Avenue, New York, NY 10016, USA
}

\begin{abstract}
In diabetic patients, vascular disease and autonomic dysfunction might compromise cerebral autoregulation and contribute to orthostatic intolerance. The aim of our study was to determine whether impaired cerebral autoregulation contributes to orthostatic intolerance during lower body negative pressure in diabetic patients.

Thirteen patients with early-stage type 2 diabetes were studied. We continuously recorded RR-interval, mean blood pressure and mean middle cerebral artery blood flow velocity at rest and during lower body negative pressure applied at -20 and $-40 \mathrm{~mm} \mathrm{Hg}$. Spectral powers of RR-interval, blood pressure and cerebral blood flow velocity were analyzed in the sympathetically mediated low (LF: 0.04-0.15 Hz) and the high (HF: $0.15-0.5 \mathrm{~Hz}$ ) frequency ranges. Cerebral autoregulation was assessed from the transfer function gain and phase shift between LF oscillations of blood pressure and cerebral blood flow velocity. In the diabetic patients, lower body negative pressure decreased the RRinterval, i.e. increased heart rate, while blood pressure and cerebral blood flow velocity decreased. Transfer function gain and phase shift remained stable.

Lower body negative pressure did not induce the normal increase in sympathetically mediated LF-powers of blood pressure and cerebral blood flow velocity in our patients indicating sympathetic dysfunction. The stable phase shift, however, suggests intact cerebral autoregulation. The dying back pathology in diabetic neuropathy may explain an earlier and greater impairment of peripheral vasomotor than cerebrovascular control, thus maintaining cerebral blood flow constant and protecting patients from symptoms of presyncope.
\end{abstract}

Keywords: Lower body negative pressure; Cerebral autoregulation; Phase shift; Transfer function gain

\section{Introduction}

Autonomic dysfunction is an important complication of diabetes mellitus. Up to $90 \%$ of diabetic patients experience autonomic-related symptoms such as impaired sweating as well as pupillary, gastrointestinal, genitourinary or cardio-

\footnotetext{
* Corresponding author. Department of Neurology, University of Erlangen-Nuremberg, Schwabachanlage 6, D-91054 Erlangen, Germany. Tel.: +49 9131853 4444; fax: +4991318534328.

E-mail address: max.hilz@neuro.imed.uni-erlangen.de (M.J. Hilz).

${ }^{1}$ Shared first authorships.
}

vascular symptoms [1,2]. Cardiovascular autonomic neuropathy is an important complication of diabetes because it is associated with increased risks of morbidity and mortality [3]. In a previous study we demonstrated that the autonomic regulation of the heart and blood vessels is impaired in patients with type 2 diabetes mellitus [4]. One manifestation of cardiovascular autonomic neuropathy in diabetic patients is orthostatic intolerance [2]. Typical symptoms of orthostatic intolerance include postural dizziness, blurred vision, neck pain or even syncope [2]. Orthostatic intolerance in patients with autonomic failure is related to an inadequate vascular resistance response to the upright posture with a subsequent drop in blood pressure [5]. There is, however, evidence that 
impaired regulation of the cerebral blood vessels might also contribute to orthostatic intolerance in some patients [6].

Cerebral autoregulation refers to the intrinsic ability of the cerebral blood vessels to maintain a near-constant cerebral blood flow despite changes in perfusion pressure, provided the blood pressure remains within certain limits $(50-150 \mathrm{~mm}$ $\mathrm{Hg}$ ). Several mechanisms are thought to contribute to cerebral autoregulation. These include myogenic, endothelial and neurogenic influences [7-9]. Diabetic patients have been shown to have endothelial dysfunction and other microvascular abnormalities [10]. It is therefore possible that in addition to dysfunctional autonomic regulation of the blood vessels, diabetic patients have impaired cerebral autoregulation.

The aim of this study was to investigate the autonomic and vascular responses to orthostatic stress in patients with type 2 diabetes mellitus. Orthostatic stress was simulated by application of lower body negative pressure (LBNP). In healthy individuals LBNP causes pooling of blood in the veins of the lower body and decreases cardiac output $[11,12]$. Blood pressure is maintained near-constant during LBNP by symphatetically mediated peripheral vasoconstriction, and there is little change in cerebral blood flow [11]. We hypothesized that patients with diabetes mellitus would have impaired regulation of the peripheral and cerebral blood vessels and that this might contribute to orthostatic intolerance in these patients.

\section{Materials and methods}

\subsection{Subjects}

Thirteen patients ( 4 women, 9 men) with diabetes mellitus type 2 were investigated. Their mean age was $58 \pm 6$ years, their height was $171 \pm 9 \mathrm{~cm}$, their weight was $84 \pm 19 \mathrm{~kg}$ and the mean duration of diabetes $85 \pm 55$ months.

All patients had been diagnosed with type 2 diabetes according to the internationally accepted recommendations based on the criteria of the American Diabetes Association and the World Health Organization (WHO) [13]. All our patients performed glycemic control. Five patients were treated with insulin and 8 patients were on oral antidiabetics such as sulfonylureas or metformin.

All participants were tested in the morning between 8 and $12 \mathrm{a}$ a.m. after an overnight fast of at least $8 \mathrm{~h}$. The diabetic patients had taken their last subcutaneous insulin injection or oral antidiabetic medication before bedtime and did not take their morning dose on the day of the study. They were instructed to avoid hypoglycemia at the day of the testing and to eat a light snack containing a cereal bar and a glass of milk if they experienced either symptoms of hypoglycemia or if their morning glucose level was below $70 \mathrm{mg} / \mathrm{dl}$. To assure the absence of hypoglycemia within $24 \mathrm{~h}$ prior to testing, all patients self-monitored their blood glucose at least three times $1 \mathrm{~h}$ after meals and reported a postprandial glucose level below $140 \mathrm{mg} / \mathrm{dl}$. To ensure long-term glycemic control, we only included patients in our study who had normal or nearnormal $\mathrm{HbA} 1 \mathrm{c}$ levels.

None of the diabetic patients showed any symptoms or signs of diabetic somatic neuropathy. One patient occasionally suffered from symptoms of orthostatic intolerance such as postural dizziness or blurred vision. One other patient had a history of diabetic retinopathy. The clinical characteristics of the patients are summarized in Table 1 .

We also tested 15 age- and sex-matched healthy controls ( 5 women, 10 men). Their mean age was $59 \pm 9$ years, their height was $171 \pm 9 \mathrm{~cm}$ and their weight was $76 \pm 16 \mathrm{~kg}$. The controls were recruited among healthy friends or relatives of staff members and patients.

The study was approved by the local ethics committee of the University of Erlangen-Nuremberg and written informed consent was obtained according to the declaration of Helsinki.

None of the participants had a history of an acute illness, chronic alcohol abuse, carcinoma of any origin, myopathy, hyper- or hypothyroidism, arterial hypertension, atherosclerosis, previous kidney or pancreas transplantation, significant hepatic or renal disease or any other condition that would interfere with the patient's ability to participate in the study. None of the participants was on any antihypertensive or other medication known to influence the cardiovascular or

Table 1

Clinical characteristics and data on diabetes control including blood glucose level reported by the patients, HbA1c, antidiabetic medication, systolic and diastolic blood pressure and diabetic complications

\begin{tabular}{|c|c|c|c|c|c|c|}
\hline Patient's ID & HbA1c $[\%]$ & Glucose level $[\mathrm{mg} / \mathrm{dl}]$ & Antidiabetic medication & Complications & Systolic BP [mm Hg] & Diastolic BP [mm Hg] \\
\hline 1 & 7.2 & 107 & Metformin & None & 132 & 78 \\
\hline 2 & 7.4 & 103 & Metformin & Retinopathy & 141 & 79 \\
\hline 3 & 6.9 & 98 & Metformin & None & 128 & 80 \\
\hline 4 & 6.7 & 97 & Metformin & None & 123 & 76 \\
\hline 5 & 7.5 & 92 & Insulin & Presyncope & 115 & 83 \\
\hline 6 & 7.3 & 111 & Insulin & None & 157 & 60 \\
\hline 7 & 6.9 & 115 & Insulin & None & 128 & 74 \\
\hline 8 & 7.7 & 105 & Glimepiride & None & 141 & 87 \\
\hline 9 & 7.5 & 99 & Metformin & None & 133 & 45 \\
\hline 10 & 7.2 & 117 & Insulin & None & 125 & 67 \\
\hline 11 & 6.8 & 101 & Metformin, glibenclamide & None & 119 & 51 \\
\hline 12 & 6.5 & 106 & Glimepiride, metformin & None & 114 & 62 \\
\hline 13 & 6.3 & 121 & Insulin & None & 132 & 77 \\
\hline
\end{tabular}


autonomic systems. Only three of the 13 patients were regularly taking lipid-lowering agents such as statins. However, these 3 patients were instructed to discontinue their medication for $18 \mathrm{~h}$ prior to the study. All participants were asked to refrain from nicotine, caffeine or alcohol $24 \mathrm{~h}$ prior to the study.

\subsection{Procedures}

Studies were performed in a quiet room with an ambient temperature of $24{ }^{\circ} \mathrm{C}$ and stable humidity. As hypo- and hyperglycemia might influence cardiovascular autonomic modulation, we only tested patients with overall good glycemic control, in whom the blood glucose level was within the normal range.

Before the testing procedure, each control and patient underwent Duplex sonographic examination of the extracranial arteries to rule out atherosclerosis and significant stenoses of the carotid arteries.

Following a period for cardiovascular stabilization, autonomic function was assessed by a standardized battery of heart rate variability (HRV) tests. This was followed by application of the LBNP protocol.

\subsubsection{Autonomic function testing}

Four tests of heart rate variability were performed and evaluated according to standard criteria: recording at rest, metronomic breathing, Valsalva maneuver and active standing up. Before each challenge maneuver an adequate period at supine rest was guaranteed to assure that heart rate (HR) had returned to baseline values. Electrocardiogram (ECG) signals were recorded, transferred to a personal computer via an analogue-digital converter and analyzed offline using an automatic program package (ProSciCard, MediSyst, Linden, Germany).

For HR analysis at rest, respiration was maintained at a frequency of 12 cycles per $\min (0.2 \mathrm{~Hz})$ to rule out any bias due to individual differences in respiration. HRV at rest was determined by calculating the mean HR, the coefficient of variation $(\mathrm{CV})$ and the root mean square of successive differences (RMSSD) from 170 successive intervals of ECG R waves. To assess sympathetic and parasympathetic influences on HR modulation, we performed a fast Fourier transformation of the HR time series recorded over $5 \mathrm{~min}$. The magnitude of HR modulation was derived from the powers of the frequency spectra in the low-frequency (LF: 0.04$0.15 \mathrm{~Hz}$ ) and high-frequency (HF: $0.15-0.5 \mathrm{~Hz}$ ) range. LF reflects sympathetic and parasympathetic influences and HF reflects the parasympathetic oscillatory influence of respiratory drive on HRV [14]. The powers in the LF and HF ranges were calculated as the integral under the LF and HF curves and expressed as $\mathrm{bpm}^{2}$ [15]. Power values below the 2.5th percentile and above the 97.5 th percentile of healthy subjects were considered abnormal.

To determine HRV during metronomic breathing, a breathing rhythm of $6 \mathrm{~s}$ inspiration and $4 \mathrm{~s}$ expiration was achieved using a bar graph displayed on a computer screen. For analysis, HR, CV and RMSSD were computed from 100 RR-intervals. In addition, we determined the minimum and maximum RR-intervals from the breathing cycle with the greatest difference between the maximum RR-interval during expiration and the minimum RR-interval during inspiration. As parameters of HRV, we calculated the difference between the maximum and minimum RR-intervals $(E-I$ difference) as well as the ratio of maximum and minimum RR-intervals ( $E-I$ ratio) [16].

The HR response to the Valsalva maneuver was recorded. We defined the Valsalva ratio as the ratio of the longest RRinterval during the first $20 \mathrm{~s}$ after blowing into a mouthpiece at a pressure of $40 \mathrm{~mm} \mathrm{Hg}$ with the glottis open for $15 \mathrm{~s}$ to the shortest RR-interval during the strain [17].

The HR response to active standing was assessed by calculating the ratio between the longest and the shortest RRinterval within the first $30 \mathrm{~s}$ after standing up (the 30:15 ratio) [14].

According to the criteria of Ziegler et al., we diagnosed cardiac autonomic neuropathy (CAN) if two or more parameters of 1.) mean HR, CV and RMSSD at rest, 2.) LF-, HFpowers at rest, 3.) mean $\mathrm{HR}, \mathrm{CV}$, RMSSD, $E-I$ difference and $E-I$ ratio during metronomic breathing, 4.) the 30:15 ratio and 5.) the Valsalva ratio exceeded age-related normal control values [16]. Based on the HRV findings, patients were classified in two subgroups (with or without CAN).

\subsubsection{LBNP protocol}

Progressive orthostatic stress was elicited by means of lower body negative pressure (LBNP). Subjects were placed in a semi-cylindrical chamber that was sealed at the level of the iliac crests. Pressure within the chamber was indicated by a manometer and could be lowered using a variable vacuum source. After a period of at least $40 \mathrm{~min}$ of quiet rest, a baseline period was recorded and LBNP then was applied at -20 and $-40 \mathrm{~mm} \mathrm{Hg}$. At each level of LBNP, we allowed at least $3 \mathrm{~min}$ for the biosignals to stabilize, then made recordings during a 3-min period of paced breathing at $0.25 \mathrm{~Hz}$ (15 breaths $/ \mathrm{min}$ ). This breathing frequency ensures that there is no interference with sympathetically mediated cardiovascular oscillations in the low-frequency $(0.04-0.15 \mathrm{~Hz})$ range. Prior to the start of the procedure, all subjects were trained in pacing their breathing at $0.25 \mathrm{~Hz}$ without hyperventilating [18].

We made continuous measurements of electrocardiogram (5-lead ECG) and noninvasive blood pressure (BP) in the radial artery at the level of the wrist by arterial tonometry (Colin Pilot ${ }^{\mathrm{TM}}$, Colin Medical Instruments Corp., San Antonio, TX, USA). Respiration was monitored by calibrated electrical inductance plethysmography (Respitrace Calibrator $^{\mathrm{TM}}$, Ambulatory Monitoring Inc., Ardsley, NY, USA). Mean cerebral blood flow velocity (CBFV) of the left proximal middle cerebral artery (MCA) was assessed by transcranial Doppler sonography (Multidop XL ${ }^{\mathrm{TM}}$, DWL, Sipplingen, Germany). The left middle cerebral artery was 
insonated at a depth of $35-55 \mathrm{~mm}$ using a 2-MHz probe, and the probe was fixed in place with a headband. Beat-to-beat values of mean velocity were recorded.

The signals of RR-interval (RRI), blood pressure, respiration and cerebral blood flow velocity were digitized at a sampling rate of $300 \mathrm{~Hz}$, fed to a computer and stored for offline analysis. A computer program identified the peak of each R wave and constructed time series of RR-interval, systolic, mean and diastolic blood pressure, mean cerebral blood flow velocities and respiration.

Oscillations in RRI, blood pressure and cerebral blood flow velocity were characterized by applying power spectral analysis to these signals using an autoregressive algorithm [19]. We identified peaks of oscillations in the low- (LF: 0.04-0.15 Hz) and high-frequency ranges (HF: 0.15$0.50 \mathrm{~Hz}$ ). As the LF- and HF-powers of RRI may be due to changes in total power, we normalized the LF- and HFpowers to more precisely quantify sympathetic and parasympathetic cardiac modulation $[15,20]$. For normalization of the powers, we calculated the percentage values by dividing the LF- or HF-powers by the sum of LF- and HFpowers and multiplying by 100 [21].

Cerebral autoregulation can be considered a high-pass filter that dampens and shifts slow BP fluctuations but allows for passing through of rapid oscillations, such as the pulsatile signals of the BP waves. We performed transfer function analysis between BP and CBFV oscillations and calculated the transfer function gain and phase shift in the LF band as parameters reflecting the quality of dynamic cerebral autoregulation. Both parameters were calculated only, if coherence between BP and CBFV was above 0.5, i.e. the two signals had a stable phase relation for a given frequency of oscillation and the signals were thought to be synchronized with each other $[20,22,23]$. We normalized the transfer function gain by dividing the LF transfer function gain by the mean values of the input signal BP and the output signal $\mathrm{CBFV}$ of cerebral autoregulation, i.e. by cerebrovascular conductance (CBFV/BP) of each patient [24].

\subsection{Statistical analysis}

All values are presented as mean \pm standard deviation (SD). Significance was assumed for $P$ values below 0.05 . As we could not assume a normal distribution, we used the nonparametric two-sided Friedman test to compare the biosignals recorded at rest and during negative pressure at -20 and $-40 \mathrm{~mm} \mathrm{Hg}$ in the healthy volunteers and in the patients. A commercially available statistical program (SPSS ${ }^{\mathrm{TM}}$, SPSS Inc., Chicago, Ill, USA) was used for data analysis.

\section{Results}

\subsection{Healthy volunteers}

None of the healthy volunteers had any pathologic finding in the heart rate variability tests.
All the healthy volunteers tolerated the LBNP procedure. In none of the controls, the LBNP protocol had to be terminated due to symptoms of orthostatic intolerance such as postural dizziness, blurred vision, neck pain or syncope.

The results of the LBNP protocol in the healthy volunteers are summarized in Table 3 .

LBNP increased HR in the controls. $\mathrm{BP}$ and $\mathrm{CBFV}$, however, remained stable during the entire LBNP protocol.

In the controls, LBNP induced a typical decrease in the parasympathetically mediated HF-power of RRI and a typical increase in the sympathetically mediated LF-power of BP. In contrast, LBNP did not influence the sympathetically mediated LF-power of CBFV in the healthy volunteers (Fig. 1).

Similarly, both parameters of cerebral autoregulation the transfer function gain and phase shift - remained stable (Fig. 2).

\subsection{Diabetic patients}

All patients adequately performed the autonomic challenge maneuvers. Assessment of heart rate at rest, during metronomic breathing, the Valsalva maneuver and active standing demonstrated significantly reduced heart rate variability in diabetics. Nine patients $(69 \%)$ were diagnosed with cardiac autonomic neuropathy (CAN) according to the
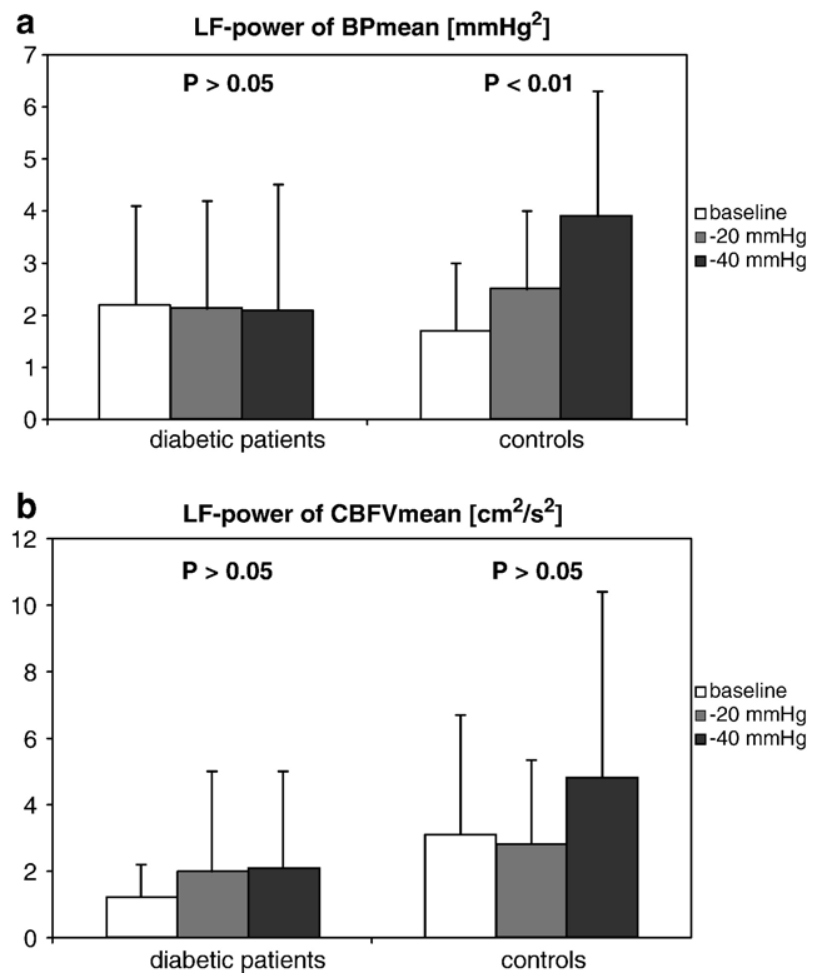

Fig. 1. Influence of lower body negative pressure on the sympathetically mediated low-frequency powers (LF-powers) of mean blood pressure $\left(\mathrm{BP}_{\text {mean }}\right)$ (a) and mean cerebral blood flow velocity $\left(\mathrm{CBFV}_{\text {mean }}\right)(\mathrm{b})$ in the 11 orthostasetolerant patients and the 15 healthy volunteers. $\mathrm{LF}$-power of $\mathrm{BP}_{\text {mean }}$ increased significantly in the controls $(P<0.01)$, but did not change significantly in the diabetics during LBNP $(P>0.05)$. LF-power of $\mathrm{CBFV}_{\text {mean }}$ did not change significantly in both groups during LBNP $(P>0.05)$. 

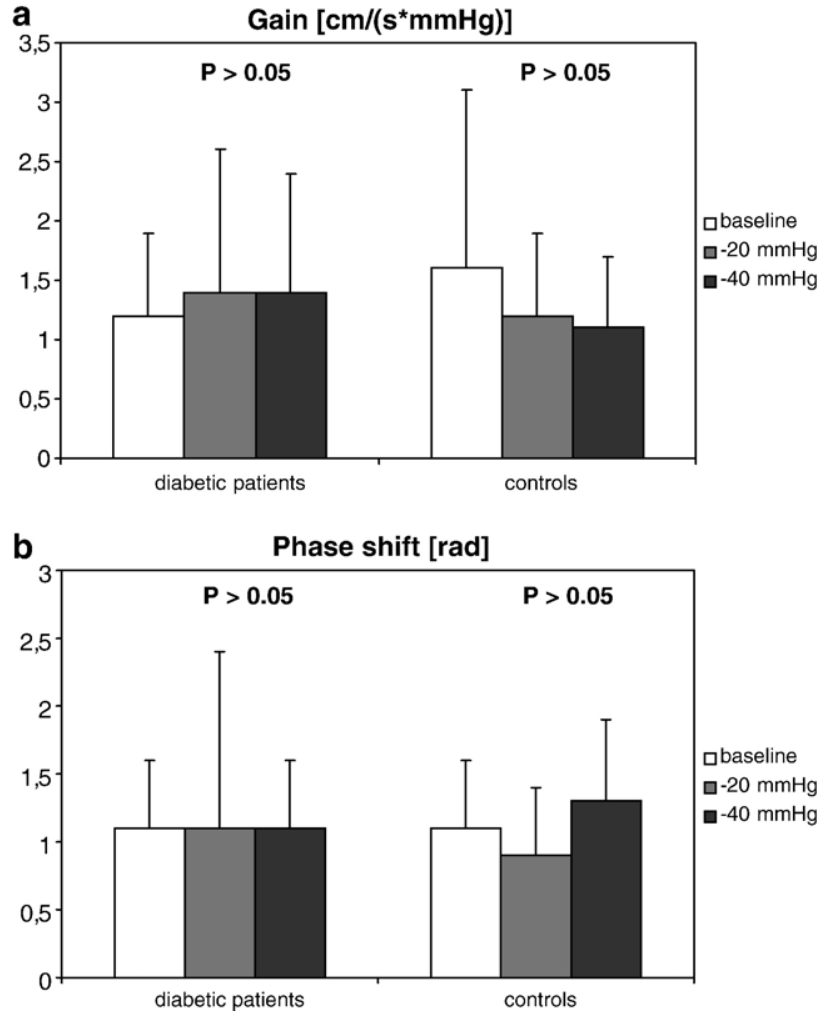

Fig. 2. Transfer function gain (a) and phase shift (b) between mean blood pressure $\left(\mathrm{BP}_{\text {mean }}\right)$ and mean cerebral blood flow velocity $\left(\mathrm{CBFV}_{\text {mean }}\right)$ oscillations in the low-frequency band in 15 healthy volunteers and the 11 orthostase-tolerant patients before and during lower body negative pressure. Transfer function gain as well as phase shift did not change significantly in both groups during lower body negative pressure $(P>0.05)$.

criteria suggested by Ziegler et al. Ten of the 13 patients (77\%) had abnormal results during metronomic breathing, 6 patients (46\%) had abnormal results during the Valsalva maneuver and 5 patients (38\%) had abnormal results during active standing (Table 2). In the remaining 4 patients $(31 \%)$, heart rate variability was within the normal range. 11 of the 13 diabetics tolerated the entire LBNP protocol. In 2 patients, the LBNP had to be terminated due to signs of presyncope. Eight of the 11 orthostatically-tolerant patients had been diagnosed with CAN.

The results of the LBNP protocol in the diabetic patients are summarized in Table 4.

As in the controls, LBNP also increased HR and tended to decrease the HF-power of RRI in the diabetic patients. In contrast to the controls, BP and CBFV significantly decreased during orthostatic challenge. The LF-powers of BP and CBFV did not change during LBNP (Fig. 1). Similarly, LBNP did not influence the transfer function gain and phase shift (Fig. 2; Table 4).

\section{Discussion}

In response to progressively applied LBNP, our healthy volunteers maintained stable blood pressure and cerebral blood flow velocity and showed the expected reflex increase in heart rate. The power of sympathetically mediated lowfrequency oscillations in mean blood pressure increased in the healthy subjects during LBNP but with no change in the power of low-frequency oscillations in cerebral blood flow velocity. These findings are consistent with previous reports [18]. In contrast, the diabetic patients showed a slight reduction in blood pressure and cerebral blood flow velocity during LBNP. The diabetics also failed to increase the power of low-frequency oscillations in blood pressure during LBNP. These results suggest that while type 2 diabetics have impaired peripheral vascular responses to orthostatic stress, there is little evidence for impaired cerebral autoregulation in these patients.

In healthy subjects blood pressure is usually maintained near-constant during orthostatic stress by arterial baroreflexmediated sympathetic vasomotor activation and cardiovagal withdrawal with a subsequent increase in heart rate $[11,20]$. The expected increase in the sympathetically mediated lowfrequency power of blood pressure fluctuations was absent from our diabetic patients and is consistent with our previous report showing impaired baroreflex-mediated peripheral vasomotor control in these patients [4]. The blood pressure reduction that we observed during LBNP in our diabetic patients was presumably due to an inadequate peripheral vasoconstrictor response.

Our diabetic patients showed an increase in heart rate during LBNP that was similar to that observed in the control subjects. According to our results from the standard heart rate variability tests, 9 of the 13 patients showed pathologic results in more than two of the cardiovascular tests including deep breathing and the Valsalva maneuver, both evaluating

Table 2

Results of the heart rate variability testing in the 15 healthy controls and the 13 patients with diabetes mellitus

\begin{tabular}{lcc}
\hline Parameter & Healthy controls & Diabetic patie \\
\hline At rest & & \\
Mean heart rate [bpm] & $67.25 \pm 12.54$ & $80.49 \pm 11.56$ \\
CV [\%] & $3.98 \pm 1.32$ & $2.14 \pm 0.80$ \\
RMSSD [ms] & $30.03 \pm 10.96$ & $7.51 \pm 2.86$ \\
LF-power & $0.49 \pm 0.37$ & $0.24 \pm 0.27$ \\
HF-power & $1.22 \pm 1.37$ & $0.16 \pm 0.16$
\end{tabular}

Metronomic breathing

Mean heart rate [bpm]

CV [\%]

RMSSD [ms]

$E-I$-difference $[\mathrm{ms}]$

$E-I$ ratio

$$
\begin{aligned}
65.81 & \pm 11.52 \\
5.50 & \pm 1.89 \\
34.74 & \pm 14.65 \\
214.22 & \pm 89.51 \\
1.26 & \pm 0.12
\end{aligned}
$$

$81.22 \pm 13.39$

$2.66 \pm 1.32$

$9.72 \pm 3.66$

$76.33 \pm 37.37$

$1.11 \pm 0.07$

Valsalva maneuver

Valsalva ratio

$$
1.34 \pm 0.20
$$

$1.24 \pm 0.19$

Active standing

30:15 ratio

$1.22 \pm 0.09$

$1.13 \pm 0.07$

(CV: coefficient of variation, RMSSD: root mean square successive differences, LF: low-frequency, HF: high-frequency). 
Table 3

Results of the LBNP protocol obtained in the 15 healthy volunteers

\begin{tabular}{|c|c|c|c|c|}
\hline Parameter & At rest & $\begin{array}{l}-20 \mathrm{~mm} \\
\mathrm{Hg} \mathrm{LBNP}\end{array}$ & $\begin{array}{l}-40 \mathrm{~mm} \\
\mathrm{Hg} \mathrm{LBNP}\end{array}$ & $\begin{array}{l}\text { Friedman } \\
\text { test }\end{array}$ \\
\hline Heart rate $[\mathrm{bpm}]$ & $62.9 \pm 9.0$ & $64.6 \pm 8.6$ & $74.3 \pm 8.2$ & $P<0.01$ \\
\hline $\begin{array}{l}\text { Normalized LF-power } \\
\text { of RRI [\%] }\end{array}$ & $42.4 \pm 25.1$ & $51.4 \pm 24.8$ & $58.7 \pm 23.5$ & $P<0.05$ \\
\hline $\begin{array}{l}\text { Normalized HF-power } \\
\text { of RRI [\%] }\end{array}$ & $76.4 \pm 30.0$ & $70.2 \pm 27.7$ & $57.4 \pm 23.5$ & $P<0.01$ \\
\hline Mean BP $[\mathrm{mm} \mathrm{Hg}]$ & $91.8 \pm 12.7$ & $93.8 \pm 17.6$ & $92.0 \pm 16.9$ & $P>0.05$ \\
\hline $\begin{array}{l}\text { LF-power of mean BP } \\
{\left[\mathrm{mm} \mathrm{Hg}^{2}\right]}\end{array}$ & $1.7 \pm 1.3$ & $2.5 \pm 1.5$ & $3.9 \pm 2.4$ & $P<0.01$ \\
\hline Mean CBFV $\left[\mathrm{cm} \mathrm{s}^{-1}\right]$ & $47.4 \pm 18.8$ & $42.5 \pm 15.1$ & $40.7 \pm 13.3$ & $P>0.05$ \\
\hline $\begin{array}{l}\text { LF-power of mean } \\
\text { CBFV }\left[\mathrm{cm}^{2} \mathrm{~s}^{-2}\right]\end{array}$ & $3.1 \pm 3.6$ & $2.8 \pm 2.5$ & $4.8 \pm 5.6$ & $P>0.05$ \\
\hline $\begin{array}{c}\text { Transfer function gain } \\
{\left[\mathrm{cm} \mathrm{s}^{-1} \mathrm{~mm} \mathrm{Hg}^{-1}\right]}\end{array}$ & $1.6 \pm 1.5$ & $1.2 \pm 0.7$ & $1.1 \pm 0.6$ & $P>0.05$ \\
\hline Phase shift [rad] & $1.1 \pm 0.5$ & $0.9 \pm 0.5$ & $1.3 \pm 0.6$ & $P>0.05$ \\
\hline
\end{tabular}

Significant differences between parameters at rest and during LBNP are indicated in bold. (LF: low-frequency, HF: high-frequency, RRI: RRinterval, BP: blood pressure, CBFV: cerebral blood flow velocity).

primarily parasympathetic function [20]. Therefore, one might expect also a reduced parasympathetic response to LBNP. While the increase in LF-power of RR-interval might not necessarily reflect sympathetic activation as the LFpower indicates both sympathetic and parasympathetic activation [15], our diabetic patients showed a decrease in the parasympathetically mediated normalized HF-power of RRinterval. Although the decrease in HF-power was not significant, there was still a shift towards a more sympathetic control of heart rate that was sufficient to induce a significant increase in heart rate during LBNP.

Although CBFV decreased slightly in the diabetic patients during LBNP, the response was similar to that observed in the control subjects. This suggests that the responses of the cerebral blood vessels are intact during orthostatic stress in diabetics. To evaluate cerebral autoregulation in more detail, we assessed the ability of the cerebral resistance vessels to buffer low-frequency blood pressure fluctuations. In the healthy volunteers, LBNP induced an increase in LF-power of BP, while the LF-power of CBFV remained stable. This finding indicates that LBNP-induced increases in LF blood pressure fluctuations were not transferred onto the cerebrovascular system, but were adequately buffered by the cerebral blood vessels. Since LBNP did not increase the LF-power of $\mathrm{BP}$ in the diabetic patients, it is not possible to determine whether this aspect of autoregulation is intact. Our results do, however, show that the application of LBNP did not induce any substantial increase in cerebral blood flow velocity fluctuations during orthostatic stress.

In addition to the transfer function gain, the phase relation between oscillations in BP and CBFV can be also used to determine the quality of cerebral autoregulation. According to Diehl et al., a decrease of the phase angle below $60-90^{\circ}$ indicates a more passive behavior of the cerebral vessel bed and is an indicator of impaired cerebral autoregulation $[20,22,24]$. In both our groups, the phase shift remained stable during LBNP confirming the assumptions of intact cerebral autoregulation during LBNP.

So far, orthostatic intolerance in patients with autonomic dysfunction has been primarily linked to impaired blood pressure regulation or to an abnormal regulation of the cerebral circulation $[6,25]$. A recent study in diabetic patients with a rather long duration of the disease showed cardiac autonomic neuropathy, orthostatic hypotension and instability in cerebral blood flow upon standing, which suggests impaired cerebral autoregulation [26]. Our patients also have an impaired sympathetic vasomotor control of peripheral blood vessels, while cerebral autoregulation still seems to be intact. In contrast to the study by Mankovsky et al. [26], our patients had diabetes at a rather early stage of the disease. In our study, peripheral vasomotor control was evaluated using blood pressure recordings at the level of the distal radial artery, while cerebral autoregulation was determined from transcranial Doppler recordings of the proximal MCA. Postganglionic sympathetic nerve fibers innervating the proximal MCA segment originate from the superior cervical ganglion and are shorter than postganglionic nerve fibers innervating the distal radial artery at the wrist with their origin in the stellate ganglion. The dying back pathology in diabetic neuropathy explains an earlier and greater impairment of peripheral vasomotor control than cerebrovascular control.

Although our patients had deficient sympathetic vasomotor control, higher levels of orthostatic stress did not induce any symptoms of orthostatic intolerance. Obviously, cerebral autoregulation still ensured stability in cerebral blood flow in the presence of sympathetic dysfunction. Long-term intensive antidiabetic treatment might be useful not only to avoid future instability of cerebral blood flow upon standing, but also to prevent for cerebral hypoperfusion with an increased risk of ischemic strokes.

Table 4

Results of the LBNP protocol obtained in the 11 patients with diabetes mellitus, who tolerated the entire LBNP protocol

\begin{tabular}{|c|c|c|c|c|}
\hline Parameter & At rest & $\begin{array}{l}-20 \mathrm{~mm} \\
\mathrm{Hg} \text { LBNP }\end{array}$ & $\begin{array}{l}-40 \mathrm{~mm} \\
\mathrm{Hg} \text { LBNP }\end{array}$ & $\begin{array}{l}\text { Friedman } \\
\text { test }\end{array}$ \\
\hline Heart rate $[\mathrm{bpm}]$ & $73.4 \pm 12.2$ & $76.0 \pm 11.5$ & $83.5 \pm 10.0$ & $P<0.01$ \\
\hline $\begin{array}{l}\text { Normalized LF-power } \\
\text { of RRI [\%] }\end{array}$ & $58.7 \pm 29.0$ & $68.4 \pm 24.0$ & $75.4 \pm 16.6$ & $P>0.05$ \\
\hline $\begin{array}{l}\text { Normalized HF-power } \\
\text { of RRI [\%] }\end{array}$ & $41.3 \pm 29.0$ & $31.6 \pm 24.0$ & $24.6 \pm 16.6$ & $P>0.05$ \\
\hline Mean BP $[\mathrm{mm} \mathrm{Hg}]$ & $89.8 \pm 10.5$ & $93.2 \pm 12.2$ & $84.6 \pm 14.4$ & $P<0.05$ \\
\hline $\begin{array}{l}\text { LF-power of mean BP } \\
{\left[\mathrm{mm} \mathrm{Hg}^{2}\right]}\end{array}$ & $2.2 \pm 1.9$ & $2.1 \pm 2.0$ & $2.1 \pm 2.4$ & $P>0.05$ \\
\hline Mean CBFV $\left[\mathrm{cm} \mathrm{s}^{-1}\right]$ & $40.8 \pm 11.6$ & $35.5 \pm 13.6$ & $34.2 \pm 13.2$ & $P<0.05$ \\
\hline $\begin{array}{l}\text { LF-power of mean } \\
\text { CBFV }\left[\mathrm{cm}^{2} \mathrm{~s}^{-2}\right]\end{array}$ & $1.2 \pm 1.0$ & $2.0 \pm 3.7$ & $2.1 \pm 2.9$ & $P>0.05$ \\
\hline $\begin{array}{l}\text { Transfer function gain } \\
{\left[\mathrm{cm} \mathrm{s}^{-1} \mathrm{~mm} \mathrm{Hg}^{-1}\right]}\end{array}$ & $0.9 \pm 0.6$ & $0.9 \pm 0.4$ & $0.8 \pm 0.4$ & $P>0.05$ \\
\hline Phase shift [rad] & $1.1 \pm 0.5$ & $1.3 \pm 0.8$ & $1.1 \pm 0.5$ & $P>0.05$ \\
\hline
\end{tabular}

Significant differences between parameters at rest and during LBNP are indicated in bold. (LF: low-frequency, HF: high-frequency, RRI: RRinterval, BP: blood pressure, CBFV: cerebral blood flow velocity). 


\section{References}

[1] Boulton AJ, Vinik AI, Arezzo JC, Bril V, Feldman EL, Freeman R, et al. Diabetic neuropathies: a statement by the American Diabetes Association. Diabetes Care 2005;28:956-62.

[2] Dutsch M, Hilz MJ, Neundorfer B. Diabetic autonomic neuropathy. Fortschr Neurol Psychiatr 2001;69:423-38.

[3] Ewing DJ, Campbell IW, Clarke BF. The natural history of diabetic autonomic neuropathy. Q J Med 1980;49:95-108.

[4] Sanya EO, Brown CM, Dutsch M, Zikeli U, Neundorfer B, Hilz MJ. Impaired cardiovagal and vasomotor responses to baroreceptor stimulation in type II diabetes mellitus. Eur J Clin Investig 2003;33:582-8.

[5] Brown CM, Stemper B, Welsch G, Brys M, Axelrod FB, Hilz MJ. Orthostatic challenge reveals impaired vascular resistance control, but normal venous pooling and capillary filtration in familial dysautonomia. Clin Sci (Lond) 2003;104:163-9.

[6] Claydon VE, Hainsworth R. Cerebral autoregulation during orthostatic stress in healthy controls and in patients with posturally related syncope. Clinic Auton Res 2003;13:321-9.

[7] Ursino M. Mechanisms of cerebral blood flow regulation. Crit Rev Biomed Eng 1991;18:255-88.

[8] Ohta K, Gotoh F, Shimazu K, Amano T, Komatsumoto S, Hamada J, et al. Locus coeruleus stimulation exerts different influences on the dynamic changes of cerebral pial and intraparenchymal vessels. Neurol Res 1991;13:164-7.

[9] Hilz MJ, Stemper B, Heckmann JG, Neundörfer B. Mechanisms of cerebral autoregulation, assessment and interpretation by means of transcranial Doppler sonography. Fortschr Neurol Neurochir Psych 2000;68:398-412.

[10] Pallas F, Larson DF. Cerebral blood flow in the diabetic patient. Perfusion 1996;11:363-70.

[11] Cooper VL, Hainsworth R. Carotid baroreceptor reflexes in humans during orthostatic stress. Exp Physiol 2001;86:677-81.

[12] Al-Shamma YMH, Hainsworth R. A quantitative comparison of the circulatory responses in humans to graded upright tilting and lower body negative pressure. In: Hainsworth R, McWilliam PN, Mary DASG, editors. Cardiogenic reflexes. Oxford: Oxford University Press; 1987. p. 431-3.

[13] Report of the expert committee on the diagnosis and classification of diabetes mellitus. Diabetes Care 2003;26(Suppl 1):S5-S20.

[14] Mathias CJ, Bannister R. Autonomic failure. A textbook of clinical disorders of the autonomic nervous system. 4 ed. Oxford: Oxford Medical Publications; 1999.
[15] Task Force of the European Society of Cardiology and the North American Society of Pacing and Electrophysiology: heart rate variability: standards of measurement, physiological interpretation, and clinical use. Circulation 1996;93:1043-65.

[16] Ziegler D, Laux G, Dannehl K, Spuler M, Muhlen H, Mayer P, et al. Assessment of cardiovascular autonomic function: age-related normal ranges and reproducibility of spectral analysis, vector analysis, and standard tests of heart rate variation and blood pressure responses. Diabet Med 1992;9:166-75.

[17] Isojarvi JI, Ansakorpi H, Suominen K, Tolonen U, Repo M, Myllyla VV. Interictal cardiovascular autonomic responses in patients with epilepsy. Epilepsia 1998;39:420-6.

[18] Brown CM, Dutsch M, Hecht MJ, Neundorfer B, Hilz MJ. Assessment of cerebrovascular and cardiovascular responses to lower body negative pressure as a test of cerebral autoregulation. J Neurol Sci 2003;208:71-8.

[19] Bernardi L, Bianchini B, Spadacini G, Leuzzi S, Valle F, Marchesi E, et al. Demonstrable cardiac reinnervation after human heart transplantation by carotid baroreflex modulation of RR interval. Circulation 1995;92:2895-903.

[20] Hilz MJ. Quantitative autonomic functional testing in clinical trials. In: Brown R, Bolton C, Aminoff M, editors. Neuromuscular function and disease. Philadelphia: W.B. Saunders Company; 2002. p. 1899-929.

[21] Pagani M, Lombardi F, Guzzetti S, Rimoldi O, Furlan R, Pizzinelli P, et al. Power spectral analysis of heart rate and arterial pressure variabilities as a marker of sympatho-vagal interaction in man and conscious dog. Circ Res 1986;59:178-93.

[22] Diehl RR, Linden D, Lucke D, Berlit P. Phase relationship between cerebral blood flow velocity and blood pressure. A clinical test of autoregulation. Stroke 1995;26:1801-4.

[23] Zhang R, Zuckerman JH, Giller CA, Levine BD. Transfer function analysis of dynamic cerebral autoregulation in humans. Am J Physiol 1998;274:H233-41

[24] Blaber AP, Bondar RL, Stein F, Dunphy PT, Moradshahi P, Kassam MS, et al. Transfer function analysis of cerebral autoregulation dynamics in autonomic failure patients. Stroke 1997;28:1686-92.

[25] Bondar RL, Dunphy PT, Moradshahi P, Kassam MS, Blaber AP, Stein $\mathrm{F}$, et al. Cerebrovascular and cardiovascular responses to graded tilt in patients with autonomic failure. Stroke 1997;28:1677-85.

[26] Mankovsky BN, Piolot R, Mankovsky OL, Ziegler D. Impairment of cerebral autoregulation in diabetic patients with cardiovascular autonomic neuropathy and orthostatic hypotension. Diabet Med $2003 ; 20: 119-26$. 